\title{
Spatially extended sound equalization in rectangular rooms
}

\section{Santillan, Arturo Orozco}

\section{Published in:}

Acoustical Society of America. Journal

Link to article, DOI:

$10.1121 / 1.1401740$

Publication date:

2001

\section{Document Version}

Publisher's PDF, also known as Version of record

Link back to DTU Orbit

Citation (APA):

Santillan, A. O. (2001). Spatially extended sound equalization in rectangular rooms. Acoustical Society of America. Journal, 110(4), 1989-1997. https://doi.org/10.1121/1.1401740

\section{General rights}

Copyright and moral rights for the publications made accessible in the public portal are retained by the authors and/or other copyright owners and it is a condition of accessing publications that users recognise and abide by the legal requirements associated with these rights.

- Users may download and print one copy of any publication from the public portal for the purpose of private study or research.

- You may not further distribute the material or use it for any profit-making activity or commercial gain

- You may freely distribute the URL identifying the publication in the public portal

If you believe that this document breaches copyright please contact us providing details, and we will remove access to the work immediately and investigate your claim 


\title{
Spatially extended sound equalization in rectangular rooms
}

\author{
Arturo O. Santillán ${ }^{\mathrm{a})}$ \\ Department of Acoustic Technology, Technical University of Denmark, Building 352, DK-2800 Lyngby, \\ Denmark
}

(Received 23 January 2001; revised 11 July 2001; accepted 12 July 2001)

\begin{abstract}
The results of a theoretical study on global sound equalization in rectangular rooms at low frequencies are presented. The zone where sound equalization can be obtained is a continuous three-dimensional region that occupies almost the complete volume of the room. It is proved that the equalization of broadband signals can be achieved by the simulation of a traveling plane wave using FIR filters. The optimal solution has been calculated following the traditional least-squares approximation, where a modeling delay has been applied to minimize reverberation. An advantage of the method is that the sound field can be estimated with sensors placed in the limits of the equalization zone. As a consequence, a free space for the listeners can be obtained. (C) 2001 Acoustical Society of America. [DOI: 10.1121/1.1401740]
\end{abstract}

PACS numbers: 43.55.Br, 43.60.Gk, 43.60.Pt [JDQ]

\section{INTRODUCTION}

The motivation of the presented study is the fact that sound reproduced in a normal listening room undergoes a spectral coloration. Since this implies a linear distortion of the reproduced sound, the effect is undesirable. Reducing the spectral coloration is particularly important at low frequencies in small enclosures, where the acoustic response of the listening space is dominated by distinct normal modes.

The equalization of the frequency response at one or several receiving points has been studied by several authors. ${ }^{1-7}$ It has been shown that these systems can achieve a high performance at the exact receiving points; however, if the distance between the target positions is relatively large, the resulting zone of equalization becomes small spheres around each control point. Moreover, the size of this region scales with the wavelength. As a consequence, the resulting system is very sensitive to changes in the position of the listener's head.

The general objective of the work described in this paper has been to study the extent to which minimizing the acoustic response of a rectangular room is possible at low frequencies in an extended and continuous three-dimensional region. The underlying idea is that this zone should be as large as possible in order to include several listeners and allow for their movements. In addition, the error detection microphones should preferably be located at unintrusive positions.

In previous theoretical and experimental studies by Santillán $^{8,9}$ the effect of the acoustic response of the room has been minimized in an extended and continuous region. In these studies it was attempted to obtain the same signal (in amplitude and phase) in the entire target zone. However, one of the conclusions was that the zone where equalization can be achieved is limited to regions with dimensions not larger than half a wavelength of the reproduced sound.

In this paper a theoretical investigation on the equalization of sound in a continuous region that occupies almost the

\footnotetext{
${ }^{a)}$ Now at: Department of Acoustics, Aalborg University, Fredrik Bajers Vej 7 B4, DK-9220 Aalborg $\emptyset$, Denmark.
}

complete volume of a rectangular room is presented. The method is based on the simulation of a plane wave moving along the room. Experimental verification of the presented method has been obtained by Santillán and Lydolf. ${ }^{10}$

\section{CONDITIONS FOR THE SIMULATION OF A TRAVELING PLANE WAVE}

Consider a rectangular room, and a Cartesian coordinate system with its axes parallel to the sides of the room and the origin in one of the lower corners. The complex sound pressure amplitude $p$ in steady state at any position $\mathbf{r}=(x, y, z)$ inside the room produced by $L$ single sources can be described by ${ }^{11}$

$$
\begin{aligned}
p(\mathbf{r})= & \frac{i \rho \omega}{V} \sum_{l=0}^{\infty} \sum_{m=0}^{\infty} \sum_{n=0}^{\infty} \frac{1}{\Lambda_{l m n}} \frac{1}{\mathbf{k}_{l m n}^{2}-k^{2}} \\
& \times \sum_{\mu=1}^{L} q_{\mu} \Psi_{l m n}\left(\mathbf{r}_{\mu}\right) \Psi_{l m n}(\mathbf{r}) .
\end{aligned}
$$

Here $\omega$ is the angular driving frequency, $\rho$ is the ambient density, $V(=L x L y L z)$ is the volume of the room, $\Psi_{l m n}$ is the mode shape, and $\mathbf{k}_{l m n}$ its complex eigenvalue, $k$ is the wave number, $\Lambda_{l m n}$ is a scaling factor, and $q_{\mu}$ is the strength of the $\mu$ th source placed at the position $\mathbf{r}_{\mu}=\left(x_{\mu}, y_{\mu}, z_{\mu}\right)$. For a lightly damped room, the modes are roughly equal to ${ }^{11}$

$$
\Psi_{l m n}(\mathbf{r})=\cos \left(l \pi x / L_{x}\right) \cos \left(m \pi y / L_{y}\right) \cos \left(n \pi z / L_{z}\right) .
$$

Assuming that the specific acoustic admittance $\beta$ is uniform over the boundaries of the room, $\mathbf{k}_{l m n}^{2}$ can be approximated to first order as

$$
\begin{aligned}
\mathbf{k}_{l m n}^{2} \simeq & \left(l \pi / L_{x}\right)^{2}+\left(m \pi / L_{y}\right)^{2}+\left(n \pi z / L_{z}\right)^{2} \\
& +2 i k \beta\left(\epsilon_{l} / L_{x}+\epsilon_{m} / L_{y}+\epsilon_{n} / L_{z}\right),
\end{aligned}
$$

where $\epsilon_{j}=2$ if $j=0$, and $\epsilon_{j}=1$ if $j>0$.

For the equalization of sound in the room, a plane wave propagating in the $y$ direction is desired. Thus under the assumption that only the axial modes in the $y$ direction are excited, Eq. (1) can be simplified as 


$$
\begin{aligned}
p(\mathbf{r})= & \frac{i \rho \omega}{V} \sum_{m=0}^{\infty} \sum_{\mu=1}^{L} \frac{1}{\Lambda_{m}} \\
& \times \frac{q_{\mu} \cos \left(m \pi y_{\mu} / L_{y}\right) \cos \left(m \pi y / L_{y}\right)}{\left(m \pi y / L_{y}\right)^{2}+2 i k \beta\left(1 / L_{x}+1 / L_{y}+1 / L z\right)-k^{2}} .
\end{aligned}
$$

With the condition that there are $N$ sound sources on the wall at $y=0$ and $L-N$ sound sources on the wall at $y=L_{y}$, and introducing $\mathbf{k}^{2}=k^{2}-2 i k \beta\left(1 / L_{x}+1 / L_{x}+1 / L_{x}\right)$, Eq. (4) can be written as

$$
\begin{aligned}
p(\mathbf{r})= & \sum_{m=0}^{\infty} \frac{\rho}{V \Lambda_{m}} \frac{-i \omega}{\mathbf{k}^{2}-\left(m \pi / L_{y}\right)^{2}} \sum_{\mu=1}^{N} q_{\mu} \cos \left(m \pi y / L_{y}\right) \\
& +\sum_{m=0}^{\infty} \frac{\rho}{V \Lambda_{m}} \frac{-i \omega}{\mathbf{k}^{2}-\left(m \pi / L_{y}\right)^{2}} \\
& \times \sum_{\alpha=N+1}^{L} q_{\alpha}(-1)^{m} \cos \left(m \pi y / L_{y}\right) \\
= & -\frac{i \omega \rho}{2 L_{x} L_{z}} \frac{1}{\mathbf{k} \sin \left(\mathbf{k} L_{y}\right)}\left[e ^ { i \mathbf { k } y } \left(\sum_{\alpha=0}^{N} q_{\alpha} e^{-i \mathbf{k} L_{y}}\right.\right. \\
& \left.+\sum_{\beta=N+1}^{L} q_{\beta}\right)+e^{-i \mathbf{k} y}\left(\sum_{\alpha=0}^{N} q_{\alpha} e^{\left.\left.i \mathbf{k} L_{y}+\sum_{\beta=N+1}^{L} q_{\beta}\right)\right],}\right.
\end{aligned}
$$

where use has been made of the following relations:

$$
\begin{aligned}
& \sum_{m=0}^{\infty} \frac{1}{\Lambda_{m}} \frac{1}{\mathbf{k}^{2}-\left(m \pi / L_{y}\right)^{2}} \cos \left(m \pi y / L_{y}\right)=L_{y} \frac{\cos \left[\mathbf{k}\left(L_{y}-y\right)\right]}{\mathbf{k} \sin \left(\mathbf{k} L_{y}\right)} \\
& \sum_{m=0}^{\infty} \frac{1}{\Lambda_{m}} \frac{(-1)^{m}}{\mathbf{k}^{2}-\left(m \pi / L_{y}\right)^{2}} \cos \left(m \pi y / L_{y}\right)=L_{y} \frac{\cos (\mathbf{k} y)}{\mathbf{k} \sin \left(\mathbf{K} L_{y}\right)}
\end{aligned}
$$

These Fourier expansions are valid from $y=0$ to $y=2 L_{y}$ for the first expression and from $y=-L_{y}$ to $y=L_{y}$ for the second one.

Since the condition $p(\mathbf{r})=P_{\alpha} e^{-i \mathbf{k} y}$, where $P_{\alpha}$ is a constant, should be satisfied to simulate a plane wave propagating in the $y$ direction, the coefficient of $e^{i \mathbf{k} y}$ in Eq. (5) has to be zero. This implies that

$$
\sum_{\beta=N+1}^{L} q_{\beta}=-e^{-i \mathbf{k} L_{y}} \sum_{\alpha=0}^{N} q_{\alpha} .
$$

From the theoretical results above, it can be seen that to simulate a plane wave traveling in the $y$ direction, only the $y$-axial modes should be excited and, according to Eq. (7), the sound sources on the wall $y=L_{y}$ should move in antiphase with the sound pressure on that wall.

On the other hand, it should be noticed that the results above are independent of the number of sound sources, the individual values of their strengths, and the position of the sources in the $x$ and $z$ coordinates on the walls at $y=0$ and $y=L_{y}$. Consequently, these factors can be used to avoid exciting the modes with $l$ or $n$ different from zero.

In a real situation, a good approximation to the conditions needed to simulate a traveling plane wave can be ob- tained at low frequencies, where relatively few modes contribute significantly to the generated sound pressure. Therefore, the excitation of the undesired modes can be reduced significantly at low frequencies by an appropriate choice of the number of loudspeakers, their position and their strength.

In the next sections, the simulation of the sound equalization in a real situation is analyzed by using a modal model of the sound field in a rectangular room. It should be observed that since only the axial $y$-modes should be excited, the sound equalization will be practically independent of the length $L_{y}$ of the room.

\section{EQUALIZATION AT SINGLE FREQUENCIES}

To analyze the fundamental physical limitations and to visualize the spatial distribution of the sound pressure level, the study of the sound equalization has been complemented in the frequency domain.

\section{A. Optimal solution}

For a given distribution of loudspeakers in the room, the optimal solution is obtained by minimizing the difference between the actual sound field that is produced in the listening zone and the desired sound field. For this purpose, the traditional least-squares approximation has been used to calculate the optimal strength of the loudspeakers. This method has been presented by Elliot et al. ${ }^{12}$ and by Asano and Swason. ${ }^{13}$ Thus following the so-called multiple-point method, the actual sound field is estimated by means of $M$ receiver positions in the listening zone. The error $e_{m}$ at the $m$ th receiving position $\mathbf{r}_{m}$ is given by

$$
e_{m}=p\left(\mathbf{r}_{m}\right)-p_{d}\left(\mathbf{r}_{m}\right)=\sum_{l=1}^{L} Z_{l}\left(\mathbf{r}_{m}\right) q_{l}-p_{d}\left(\mathbf{r}_{m}\right),
$$

where the complex amplitude of the actual sound pressure $p\left(\mathbf{r}_{m}\right)$ at the receiver position $m$ has been expressed in terms of the individual strength $q_{l}$ of each of the $L$ sound sources and the transfer impedances $Z_{l}$ from the $l$ th source to the $m$ th receiving point; $p_{d}\left(\mathbf{r}_{m}\right)$ is the desired complex sound pressure at that point.

To obtain the best solution for the sound equalization, the cost function

$$
J=\frac{1}{M} \sum_{m=1}^{M}\left|e_{m}\right|^{2}=\frac{1}{M} \sum_{m=1}^{M}\left|p\left(\mathbf{r}_{m}\right)-p_{d}\left(\mathbf{r}_{m}\right)\right|^{2}
$$

is minimized. Thus this cost function can be expressed in a quadratic form in terms of the individual strengths of the $L$ sound sources. It has been shown that the cost function has a unique global minimum, which corresponds to the optimal solution.

\section{B. Conditions for the simulations}

A room with dimensions $L_{x}=2.7 \mathrm{~m}, L_{y}=5.0 \mathrm{~m}$, and $L_{z}=2.5 \mathrm{~m}$ has been considered for the example presented in this paper. All the modes, axial, tangential, and oblique, with a natural frequency up to $1000 \mathrm{~Hz}$ (3846 modes) were used to simulate the sound pressure. The damping ratio was as- 


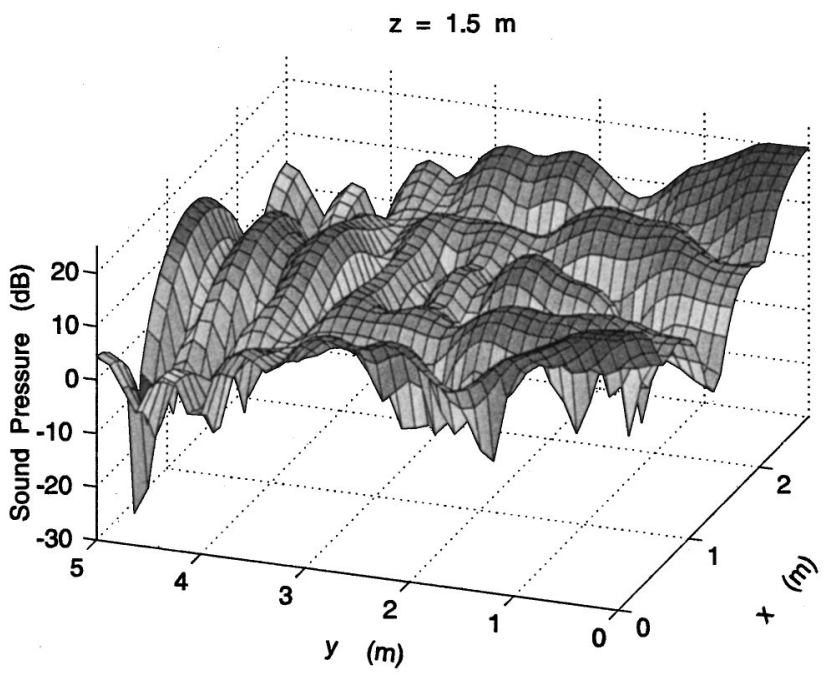

FIG. 1. Amplitude of the sound pressure before equalization for a driving frequency of $300 \mathrm{~Hz}$. The graph corresponds to the sound field in the plane $z=1.5 \mathrm{~m}$ produced by two loudspeakers placed at $(0.05,0,2.00)$ and $(2.65,0,2.00) \mathrm{m}$, respectively.

sumed equal to 0.03 for all the modes; this value corresponds to a reverberation time of approximately $0.2 \mathrm{~s}$ at frequencies around $180 \mathrm{~Hz}$. The loudspeakers were modeled as square pistons with a side length of $0.1 \mathrm{~m}$. The value of the sound speed was $346.4 \mathrm{~m} / \mathrm{s}$, for an ambient temperature of $25^{\circ} \mathrm{C}$.

\section{Distribution of loudspeakers}

It was found out that in order to excite mainly the axial modes in the $y$ direction and reduce significantly the amplitude of the other modes, the loudspeakers should be placed equidistantly in the $x$ and the $z$ directions on each of the two walls perpendicular to the $y$ axis. In addition, to minimize the reflection of sound on the surface at $y=L_{y}$, a condition expressed by Eq. (7), the same number of loudspeakers should be used in each of those two walls.

For the simulations shown below, a number of 16 loudspeakers were placed on each wall perpendicular to the $y$ axis. The 16 loudspeakers in each set were distributed in 4 horizontal lines at the heights $z=0.05 \mathrm{~m}, L_{z} / 3,2 L_{z} / 3$, and $L_{z}-0.05 \mathrm{~m}$, respectively; four loudspeakers in each horizontal line with their respective centers at the coordinates $x$ $=0.05 \mathrm{~m}, L_{x} / 3,2 L_{x} / 3$, and $L_{x}-0.05 \mathrm{~m}$.

\section{Spatial distributions of the sound field}

The spatial distributions of the sound pressure amplitude in the enclosure before and after equalization for a pure tone of $300 \mathrm{~Hz}$ are presented as an example. Before equalization, the sound field in the room was assumed to be produced by two loudspeakers fed with the same input signal. The positions of these loudspeakers are, respectively, $(0.05,0,2.00) \mathrm{m}$ and $(2.65,0,2.00) \mathrm{m}$. The amplitude of the sound field in the plane $z=1.5 \mathrm{~m}$ before equalization is shown in Fig. 1. It can be seen that the sound pressure amplitude depends on the position inside the room. The graph of Fig. 1 can be compared with the distribution of the sound pressure amplitude in the same plane after sound equalization, which is depicted in Fig. 2. It can be observed in this figure that the sound

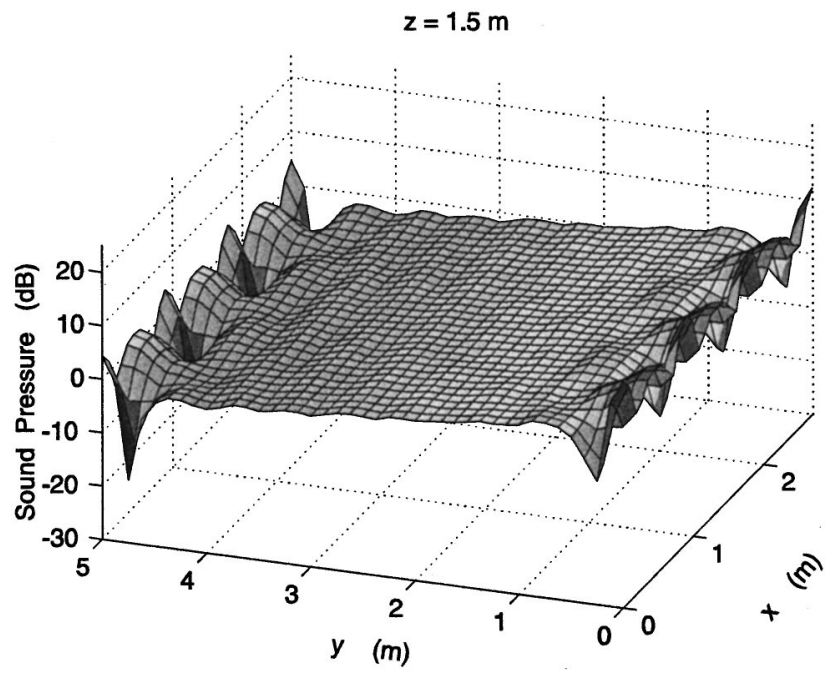

FIG. 2. Optimal equalization in the frequency domain for a driving frequency of $300 \mathrm{~Hz}$. The sound pressure level in the plane $z=1.5 \mathrm{~m}$ is shown.

pressure amplitude is almost constant in nearly the entire plane with exception of the region close to the walls perpendicular to the $y$ direction. The deviations of the obtained sound pressure amplitude from the desired value were relatively high within a distance of about $0.6 \mathrm{~m}$ from the sound sources though. It was found out that this distance of about $0.6 \mathrm{~m}$ where a good sound equalization cannot be achieved is practically independent of the reproduced frequency; therefore, the listening zone was assumed as the region from 0.6 $\mathrm{m}$ up to $4.4 \mathrm{~m}$ in the $y$ direction that occupies all the width $L_{x}$ of the room and from the floor to the ceiling. The sensor positions for the calculation of the optimal equalization were placed only inside the listening zone equidistantly distributed. The number of these sensor positions was $7 \times 12 \times 7$ (588 in total); this means that the sensor positions were arranged in 7 horizontal planes, with 12 lines parallel to the $x$ direction in each plane, and 7 sensors in each of these lines. A good estimation of the sound field can be obtained with fewer sensor positions; however, such a number was used to reduce the error in the estimation of the sound field as much as possible. The amplitude of the desired sound pressure was set to unity at each sensor position; the phase depended on the position in the $y$ direction of the error sensor corresponding with a plane traveling wave.

It should be mentioned that after equalization the sound pressure level in the listening zone was practically independent of the $z$ direction; the spatial distribution of the sound field in any plane $x y$ was basically the same as the one shown in Fig. 2.

\section{E. Values of the least-squares error}

To evaluate the results of the sound equalization, the square root of the minimum value of the cost function, given by Eq. (9), has been used. In what follows, this parameter is called the least-squares error $\left(E_{\mathrm{LS}}\right)$. It was observed that if the value of $E_{\mathrm{LS}}$ was less than 0.3 in the presented example, the deviations of the sound pressure from the desired value were within $\pm 3 \mathrm{~dB}$ in almost the entire listening zone. Thus the condition $E_{\mathrm{LS}}<0.3$ has been used here as a criterion for 


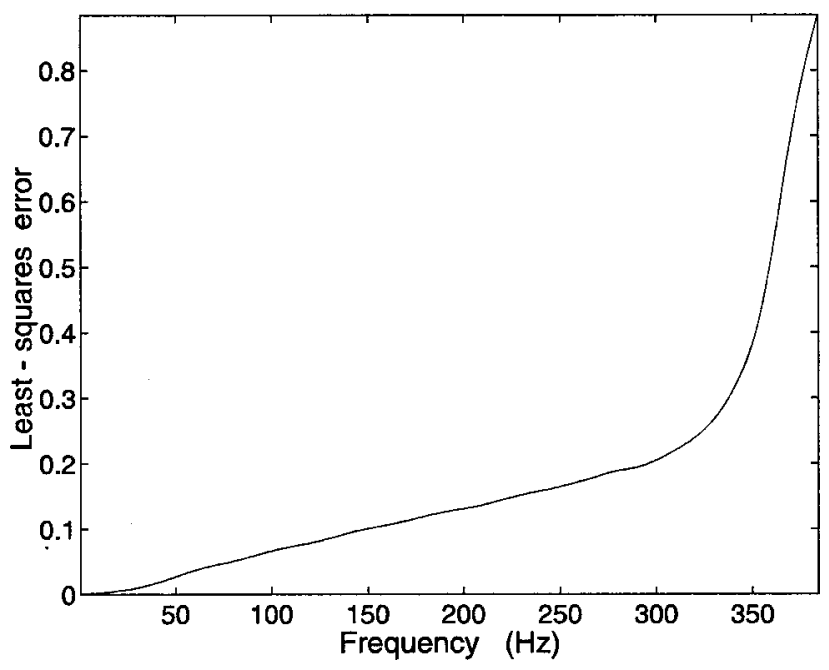

FIG. 3. The least-squares error as a function of the driving frequency for the sound equalization by simulating a plane wave moving in the $y$ direction.

a good sound equalization. The least-squares error as a function of the frequency for the considered example is shown in Fig. 3. It can be seen in this figure that the least-squares error increases with increasing frequency, and that the maximum frequency that can be equalized properly is around $343 \mathrm{~Hz}$.

\section{SOUND EQUALIZATION OF RANDOM SIGNALS}

Since echoes are undesirable in the reproduction of transient signals, the duration of the impulse response in the listening zone should be minimized. For this reason a modeling delay has been used to approximate the impulse response in the listening zone to a delayed delta function. ${ }^{14}$ As a plane wave traveling in the $y$ direction is desired to be simulated, the length of the delay depends on the ordinate of the listening position corresponding with the propagation of the plane wave.

\section{A. Optimal solution}

A matrix formulation for the reproduction of sound in a least-squares sense has been developed by Nelson et al.; ${ }^{4,14}$ this formulation has been followed for the equalization of broadband signals in the work presented in this paper. A block diagram of the equalization problem is depicted in Fig. 4. Here the input signal is reproduced by using $L$ loudspeakers. The sound field in the listening zone is represented by $M$ sample signals measured with $M$ microphones. The aim is to design $L$ digital FIR filters with impulse responses $h_{l}(n)$, one for each sound source, such that the obtainable signal $d_{m}^{\prime}(n)$ at the microphone $m$ is the best approximation to the

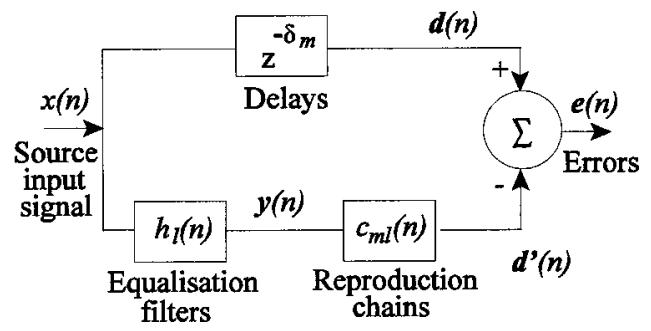

FIG. 4. Block diagram of the equalization problem of broadband signals. desired signal $d_{m}(n)$ at that sensor. Here $d_{m}(n)$ is a delayed version ( $\delta_{m}$ samples) of the original input signal $x(n)$ (modeling delay). This procedure has been discussed by Elliott et $a .^{2}{ }^{2}$ In Fig. $4, \mathbf{y}(n)$ is the vector of input signals to the $L$ sound sources, $\mathbf{d}^{\prime}(n)$ is the vector of the $M$ actual signals at each of the microphones, $\mathbf{d}(n)$ is the vector of the $M$ desired signals, and $c_{m l}(n)$ is the electroacoustic impulse response from the input to the $l$ th source to the output of the $m$ th sensor. It is assumed that these impulse responses can be accurately modeled as FIR digital filters with $J$ coefficients. In addition, the equalization filters will have a FIR structure as well with $I$ coefficients.

The optimal coefficients of these filters are determined by minimizing a performance index defined $b^{2,12}$

$$
\Gamma=E\left\{\sum_{m=1}^{M} e_{m}(n)^{2}\right\}=E\left\{\mathbf{e}(n)^{T} \mathbf{e}(n)\right\},
$$

where $E$ represents the expectation operator, and $e_{m}(n)$ $=d_{m}(n)-d_{m}^{\prime}(n)$. It has been shown that the performance index can be expressed as a quadratic function in terms of all the individual coefficients in the equalization filters. It has also been concluded that it has a unique global minimum value, which corresponds to the optimal control filter coefficients.

\section{B. Conditions for the simulations}

It should be noticed that by minimizing the performance error defined in Eq. (10), the optimal solution in statistical sense is calculated. Thus considering that the desired signal is a delayed version of the source input signal, for the described method the only required information on the source input signal is the autocorrelation function. In the simulations that were carried out, an input signal with a delta function as the autocorrelation function has been assumed. It should be noticed, however, that the input signal has to be low-filtered in order to avoid the aliasing effect. For the simulations, the sampling frequency was equal to $770 \mathrm{~Hz}$. The loudspeakers were modeled as first-order analog high pass filters with one pole at $100 \mathrm{~Hz}$.

The classical modal reconstruction model was used to calculate the discrete electroacoustic impulse responses $c_{m l}(n)$. The conditions for the computer simulations are the same described in Sec. II B and in Sec. II C. First, the frequency response functions from the input to each loudspeaker to the output of each sensor were calculated with the modal model. These frequency response functions were multiplied by the frequency response of the antialiasing filter and by the frequency response of the loudspeakers. Eventually, an inverse FFT was applied to obtain the desired discrete impulse responses $c_{m l}(n)$. An FIR filter with 400 coefficients was used to represent each of the impulse responses $c_{m l}(n)$. It was observed that the results of the equalization were highly dependent on the minimization of the aliasing effect. Therefore, a 14th-order analog low pass Butterworth filter with a cutoff frequency of $260 \mathrm{~Hz}$ was applied.

It has been found out that the best simulation of the plane wave moving across the room was obtained by placing the error sensors in two planes perpendicular to the direction 


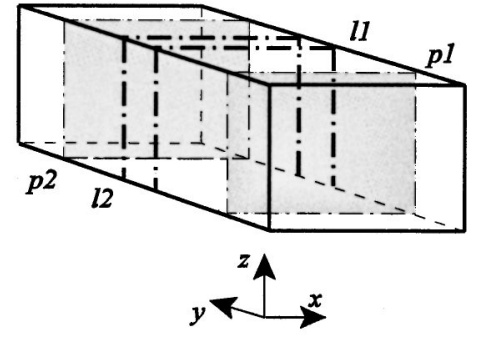

(a)

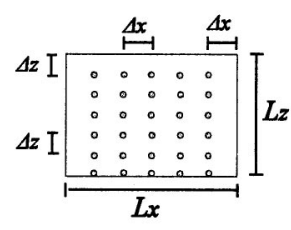

(b)

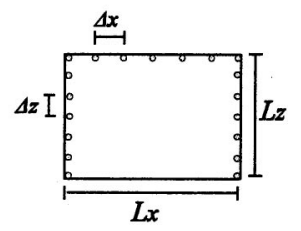

(c)

FIG. 5. Diagram of the distribution of the error sensors. These sensors were placed in two planes, indicated by $p 1$ and $p 2$ in (a), and along two lines on the walls and the ceiling of the room, denoted by $l l$ and $l 2$. In each plane there were 30 sensors arranged as shown in (b). The distribution of the sensors along the lines is depicted in (c). The distance between two adjacent sensors were $\Delta x=0.45 \mathrm{~m}$ and $\Delta z=0.41 \mathrm{~m}$.

of propagation of this wave. These two planes should be separated from each other by the distance that the sound travels during one sampling period. In addition, the error in the simulation is reduced if these planes are located close to the middle of the room.

The reason to place the sensor positions very close to each other in the direction of propagation of the simulated wave is the following one: the simulation of a traveling plane wave cannot be carried out appropriately if the distance between two error sensor positions in the direction of propagation of the plane wave is equal to multiples of half a wavelength of the sound to be simulated. In this case the delays applied in the original input signal to obtain the desired signals at those sensors will produce a difference in phase between those two desired signals equal to multiples of pi radians. However, this difference in phase is the same as the one that corresponds to a stationary wave at the two sensor positions. As a consequence, a standing wave pattern will result in the obtained sound field at those frequencies.

Since a free listening zone was desired, the sensor positions should be placed as close as possible to the limits of the listening zone. An appropriate distribution that corresponds to the example presented in the paper is as follows: 19 sensors placed in each of two lines parallel to each other on the walls and the ceiling, one line at $y=2.73 \mathrm{~m}$ and the other at $y=3.18 \mathrm{~m}$. In addition, 30 sensors placed in a plane at $y$ $=0.93 \mathrm{~m}$ and other 30 sensors on a plane at $y=4.08 \mathrm{~m}$. A diagram illustrating the distribution of these 98 sensor positions is shown in Fig. 5. The distances between to adjacent sensors were equal to $0.45 \mathrm{~m}$ in the $x$ direction and $0.41 \mathrm{~m}$ in the $z$ direction. It was found that, in general, the distance in the $x$ and in the $z$ directions between two adjacent microphones should be less than half a wavelength of the frequency to be equalized. It should be pointed out that an alternative to reduce the number of the error sensors was not

analyzed. A possibility is to consider the symmetries in the problem.

A delay of 18 samples was applied to obtain the desired signal for all the sensors placed in the plane at $y=0.93 \mathrm{~m}$. Additional delays of four, five, and seven samples were used for the sensors in the two lines in the middle of the room and in the second plane. These additional delays are equal to the time that the simulated plane wave should take to travel from the plane $y=0.93 \mathrm{~m}$ to reach the corresponding error sensor positions.

\section{Impulse and frequency responses before and after equalization}

The impulse and frequency responses at four positions in the listening zone before and after equalization are shown in Fig. 6. In this case the sound equalization was carried out with the error sensor positions placed in the limits of the listening zone as described above. The original impulse and frequency responses include the effect of the antialiasing filter, and the impulse responses after equalization are shown with an offset of -2 units in the $y$ axis for clarity. These four positions are different from the locations of the error sensors. Before equalization the sound field was assumed to be produced by two loudspeakers fed with the same input signal and placed, respectively, at the positions $(0.05,0,2.0) \mathrm{m}$ and $(2.65,0,2.0) \mathrm{m}$, as in the previous sections. A number of 100 coefficients were used in each of the control filters.

It can be seen in Fig. 6 that the frequency responses have been significantly improved after equalization. The peaks and troughs in the curves without equalization were practically removed after applying the control filters. Thus the frequency responses became approximately flat from about $20 \mathrm{~Hz}$ to approximately $340 \mathrm{~Hz}$. In this frequency interval, the deviations from the desired value of $0 \mathrm{~dB}$ are within approximately $\pm 3 \mathrm{~dB}$. It should be noticed that the peaks and troughs in the frequency responses that are not common to all the different error sensor positions can be successfully suppressed, in contrast to the case of sound equalization at discrete points, where only the common peaks and troughs can be removed. It can also be observed in the graphs of Fig. 6 that the impulse responses approximate quite well a delayed delta function. The echoes have been reduced significantly. It can be seen that the delays in the obtained impulse responses after equalization are not the same for the shown positions, but the delay depends on the value of the ordinate of the position according to the simulated plane progressive wave. It should be mentioned that the results at any other position in the listening zone are very similar to the ones shown in Fig. 6.

\section{Results of the sound equalization in terms of the least-squares error}

To evaluate the results of the sound equalization in terms of the frequency and the spatial distribution of the sound pressure in the listening zone, the impulse responses of the optimal control filters that produced the results of Fig. 6 were transformed to the frequency domain. Thus the frequency responses of the optimal filters were used in Eq. (9) to cal- 

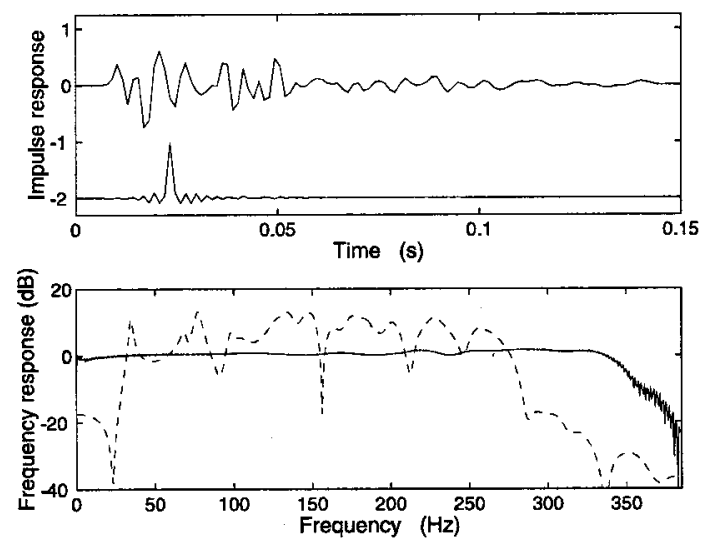

( a )
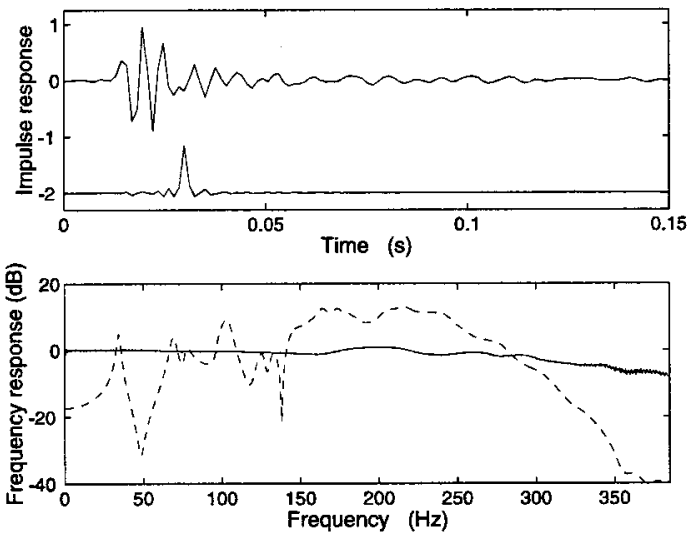

(c)
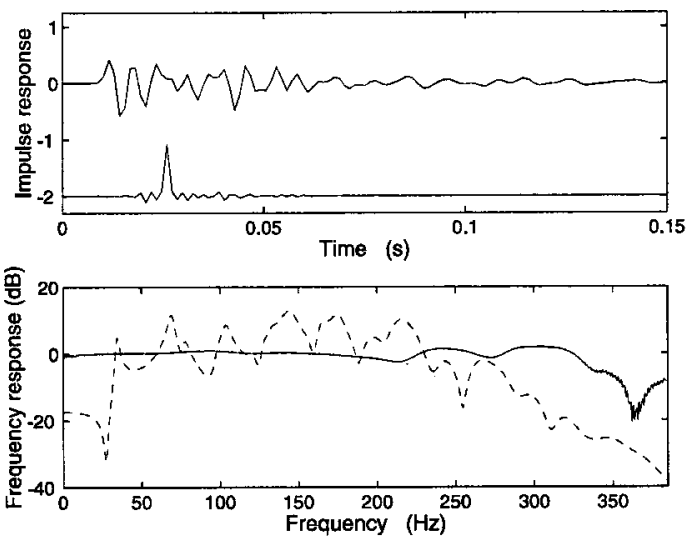

(b)
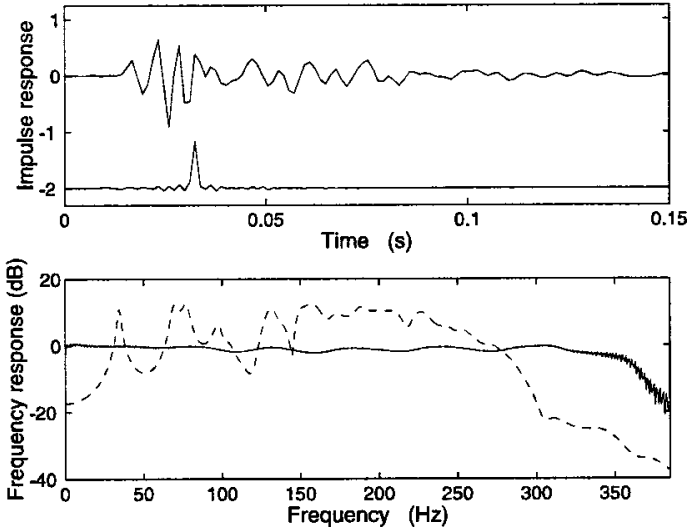

(d)

FIG. 6. Impulse and frequency responses at four different positions before and after sound equalization, (a) $(0.3,0.9,0.3) \mathrm{m},(\mathrm{b})(1.0,1.8,0.9) \mathrm{m},(\mathrm{c})(1.7,3.2,1.5)$ $\mathrm{m}$, (d) $(2.4,4.1,2.2) \mathrm{m}$. The impulse response after equalization is shown with an offset of minus two units in the $y$ axis for clarity. The solid lines in the frequency response represent the results after equalization and the dashed lines correspond to the results before equalization, which include the response of the antialiasing filters.

culate the least-squares error as a function of the frequency. In this calculation only the amplitude of the sound pressure was taken into account; the error at the $m$ th receiver was defined as $e_{m}=\left|p\left(\mathbf{r}_{m}\right)\right|-\left|p_{d}\left(\mathbf{r}_{m}\right)\right|$ instead of Eq. (8). A number of $7 \times 12 \times 7$ sensor positions in listening zone were used to approximate the sound field in the complete threedimensional region. The resulting cost function is depicted in Fig. 7, where the curve of Fig. 3, which corresponds to the equalization in the frequency domain, is included for comparison. The sound equalization was also carried out with the error sensor positions placed in two parallel planes in the middle of the room, 49 sensor positions in each plane. The graph of the least-squares error calculated for this case is shown as well in Fig. 7.

As it can be seen in Fig. 7, the results of sound equalization obtained with the error sensors placed in two planes in the middle of the room are very similar to the results of the equalization carried out in the frequency domain for frequencies higher than approximately $20 \mathrm{~Hz}$. The least-squares error was higher with the error sensor positions near the limits of the listening zone. Two are the main causes for the higher error in this case: (1) a part of the effort of the control filters is used on trying to reduce the error at these sensor positions at the expense of degrading the sound field in the rest of the listening zone; (2) the sound field cannot be estimated properly around certain frequencies due to the distribution of the error sensors as mentioned in Sec. IV B. The second reason contributes more to the relatively high values of the least-squares error at the frequencies around $110 \mathrm{~Hz}$, $165 \mathrm{~Hz}$, and $220 \mathrm{~Hz}$. Here the distance between the two planes with the error sensor positions $(4.08 \mathrm{~m}-0.93 \mathrm{~m})$ is equal to multiples of half a wavelength at those frequencies.

It might be worth placing the error sensors in the limits of the equalization zone. An adaptive algorithm can be implemented since the error sensors are unobtrusive for the listeners. For a particular application, it should be investigated whether it is advantageous to implement the system adaptively. If only a single adjustment during the installation of the audio system is sufficient to achieve a good equalization and then the control filters are held fixed, the obvious position of the error sensors will be in two planes in the middle of the enclosure.

According to Fig. 7, the equalization of the broadband signal is affected at frequencies lower than approximately 15 $\mathrm{Hz}$ in comparison to the results from the frequency domain. The reason might be the assumed poor response of the loudspeakers at that frequency range. However, this is not a problem in practice since these frequencies are not audible. 


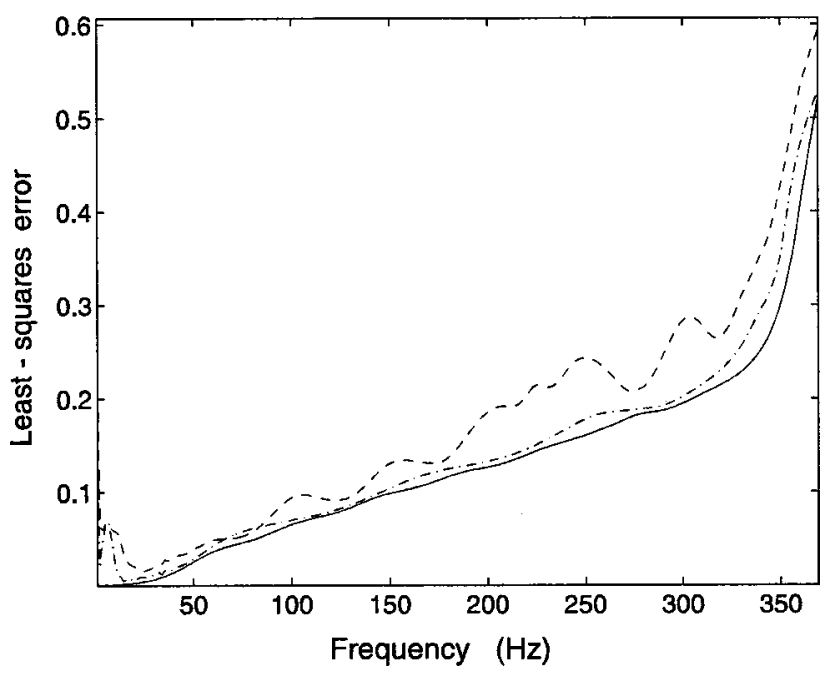

FIG. 7. Least-squares error as a function of the frequency for the sound equalization carried out in the time domain with the sensors placed near the limits of the listening zone (--) and the sensors placed in the middle of that zone (-.-). The results obtained in the frequency domain are shown for comparison (solid line).

The highest value of the least-squares error in the case where the error sensors were placed near the limits of the equalization zone occurred approximately at $300 \mathrm{~Hz}$ as can be seen in Fig. 7. Here the least-squares error is equal to 0.29 , which is slightly lower than the considered limit of the criterion for a good equalization. The distribution of the sound pressure amplitude in the plane $z=1.5 \mathrm{~m}$ for a driving frequency of $300 \mathrm{~Hz}$ after the equalization with the sensor positions in the limits of the listening zone is depicted in Fig. 8. It can be noticed that several dips in the spatial distribution of the sound pressure level appeared along the $y$ direction for this particular frequency. The distance between two adjacent dips is equal to half a wavelength, and therefore, this suggests that the reflection of acoustic energy on the wall at the end of the room was not sufficiently reduced. A very

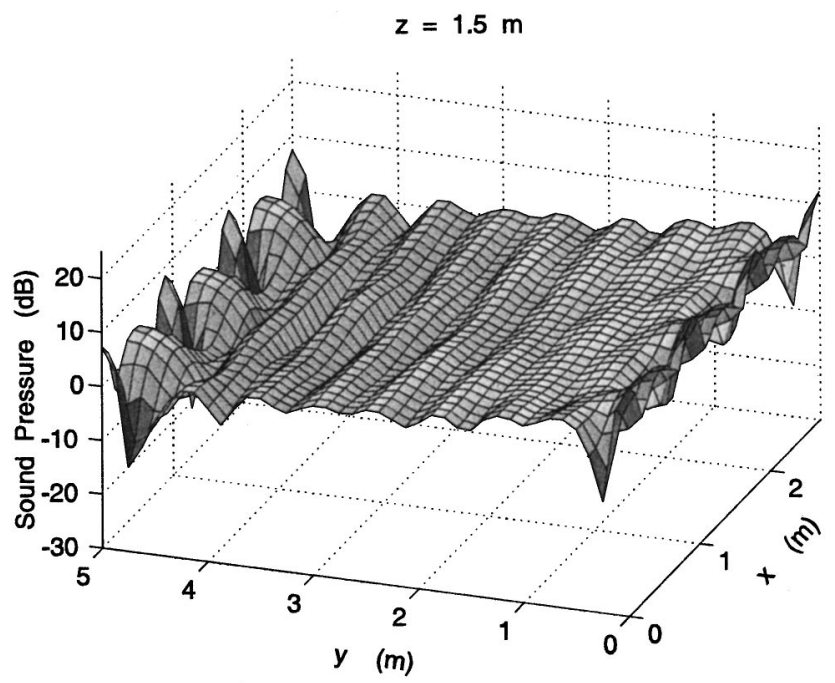

FIG. 8. Distribution of the sound pressure level at the frequency of $300 \mathrm{~Hz}$ after sound equalization in the time domain using random noise as the input signal. The result is shown in the plane $z=1.5 \mathrm{~m}$.

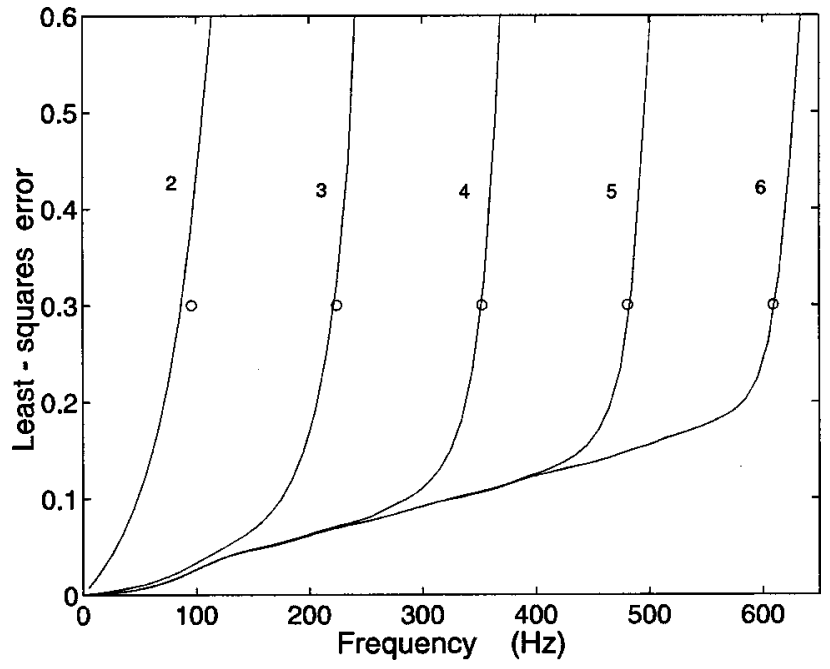

FIG. 9. Least-squares error as a function of the frequency corresponding to different numbers of loudspeakers used in the sound equalization in the frequency domain. The numbers in the figure indicate the total of loudspeakers placed on the wall at $y=0$, and the small circles are predicted values calculated from the distance in the $x$ direction between adjacent sound sources.

similar graph is obtained if another plane $x y$ is chosen instead of $z=1.5 \mathrm{~m}$.

The graph of Fig. 8 can be compared with the graph of the spatial distribution of the sound pressure amplitude at the same frequency before equalization, which is shown in Fig. 1. It can be observed that the spatial distribution of the sound field has been considerably improved after equalization using a broadband signal.

\section{NUMBER OF LOUDSPEAKERS AND THE CONTROL OF MODES}

To discuss the relation between the number of loudspeakers and the maximum frequency that can be equalized, a two-dimensional problem is considered first for simplicity. An enclosure with dimensions $L_{x}=2.7 \mathrm{~m}, L_{y}=3.0 \mathrm{~m}$, and $L_{z}=0.15 \mathrm{~m}$ has been used, and a plane wave moving in the $y$ direction has been simulated.

It was found out that, as a first approximation, the maximum frequency that can be equalized is given by

$$
f_{\max }=c / d-\Delta_{\epsilon},
$$

where $c$ is the sound speed, $d$ is the distance in the $x$ direction between two adjacent loudspeakers, and $\Delta_{\epsilon}$ is a number that depends on the damping of the room.

The curves of the least-squares error corresponding to different numbers of loudspeakers are plotted as a function of the driving frequency in Fig. 9. The number close to each curve indicates the total of sound sources placed on the wall at $y=0$. The curves in this figure were obtained by minimizing the cost function of Eq. (9) in the frequency domain. A number of $8 \times 15 \times 3$ error sensor positions within the equalization zone were used. With the criterion $E_{\mathrm{LS}}<0.3$, the value of $\Delta_{\epsilon}$ was determined to be equal to $32 \mathrm{~Hz}$. In this way, the frequencies given by $f_{\max }=c / d-32 \mathrm{~Hz}$ for the different numbers of loudspeakers are plotted by circles in Fig. 9. It can be seen in this figure that each of these circles are 
very close to the point in the corresponding curve for which $E_{\mathrm{LS}}=0.3$. Thus there is a good agreement between the value of the maximum frequency that can be equalized obtained by minimizing the cost function and the value calculated by using $f_{\max }=c / d-32 \mathrm{~Hz}$.

With the used arrangement of loudspeakers, the filtered signals fed to each of the sound sources at $y=0$ are practically in phase to each other. This fact together with Eq. (11) indicate that the mode with a wavelength equal to the distance between two adjacent sound sources is the mode with $l \neq 0$ whose excitation cannot be reduced significantly and that has the lowest frequency. In this case the loudspeakers are placed at the positions where the oscillations of the mode have the maximum amplitude and the same phase. Thus the loudspeakers are coupled very efficiently to that mode. When the difference between the driving frequency and the frequency of the mode is less than $\Delta_{\epsilon}$, the contribution of this mode becomes significant.

An analog situation occurs for a three-dimensional case. The results of the sound equalization in the vertical direction are mainly affected by the modes with $n \neq 0$ that contribute significantly to the produced sound field. Thus the mode with $n \neq 0$ that has the lowest frequency and whose excitation cannot be reduced appropriately corresponds to a wavelength equal to the distance in the $z$ direction between two adjacent sound sources.

For the simulations presented in the previous sections, the value of $\Delta_{\epsilon}$ is $40 \mathrm{~Hz}$. Since the distance between two adjacent loudspeakers in the $x$ direction $(0.90 \mathrm{~m})$ was larger than the distance in the $z$ direction $(0.83 \mathrm{~m})$, the maximum frequency that can be equalized according to Eq. (11) is $f_{\max }=c / 0.90 \mathrm{~m}-40 \mathrm{~Hz}=345 \mathrm{~Hz}$.

\section{DISCUSSION}

It has been shown that the simulation of a progressive plane wave moving along a rectangular room is possible. If all the walls are covered with loudspeakers, the presented method can be adapted to simulate plane waves in an arbitrary horizontal direction. Moreover, the simulation of cylindrical waves might be possible as well. Thus the method can be considered as an implementation of wave field synthesis. However, the system will become more complicated since more loudspeakers are required and more error sensor positions will probably be needed.

The possibility to simulate plane waves moving in any desired horizontal direction and cylindrical waves in a rectangular room has been shown theoretically based on computer simulations in the frequency domain by Santillán et al. ${ }^{15}$

It should be mentioned that the purpose in the research described in the paper has not been as demanding as to give the listener the perception of direction, but to compensate for the undesired spectral coloration and reverberation in an extended region inside rectangular rooms at low frequencies.

\section{CONCLUSIONS}

According to the results presented in this paper, it can be concluded that a good sound equalization at low frequencies can be achieved by the simulation of a traveling plane wave in a rectangular room. The sound equalization can be obtained in a continuous three-dimensional region that occupies almost the complete volume of the room. It has been proved that the equalization of random signals is possible, and that the duration of the impulse response in the listening zone can be reduced significantly. In this way, the impulse response at any point in this zone can be approximated to a delayed delta function.

It has also been shown that the equalization of broadband signals can be achieved with the error sensors placed near the limits of the equalization zone. As a consequence, a free space for the listeners and their movements is obtained.

The number of loudspeakers that are needed for the studied method of sound equalization is, however, relatively large. Since the maximum frequency that can be equalized depends on the distance between two adjacent loudspeakers, more sound sources are required for the equalization of higher frequencies or larger rooms.

\section{ACKNOWLEDGMENTS}

The author would like to thank Professor Finn Jacobsen for a number of very useful discussions and suggestions. The work was supported by the Dirección General de Asuntos del Personal Académico, Universidad Nacional Autónoma de México.

\footnotetext{
${ }^{1}$ M. Miyoshi and Y. Kaneda, "Inverse filtering of room acoustics," IEEE Trans. Acoust., Speech, Signal Process. 36, 145-152 (1988).

${ }^{2}$ S. J. Elliott and P. A. Nelson, "Multiple-point equalization in a room using adaptive digital filters," J. Audio Eng. Soc. 37, 899-907 (1989).

${ }^{3}$ S. J. Elliott, L. P. Bathia, F. S. Deghan, A. H. Fu, M. S. Stewart, and D. W. Wilson, "Practical implementation of low-frequency equalization using adaptive digital filters," J. Audio Eng. Soc. 42, 988-998 (1994).

${ }^{4}$ P. A. Nelson, F. Orduña-Bustamante, and H. Hamada, "Inverse filter design and equalization zones in multichannel sound reproduction," IEEE Trans. Speech Audio Process. 3, 185-192 (1995).

${ }^{5} \mathrm{R}$. Walker, "Equalization of room acoustics and adaptive systems in the equalization of small room acoustics," Proceedings of AES 15th International Conference, Audio, Acoustics \& Small Spaces, October 31November 2, 1998, Snekkersten, Copenhagen, Denmark (Audio Engineering Society, 1998), pp. 32-47.

${ }^{6}$ O. Kirkeby and P. A. Nelson, "Digital filter design for inversion problems in sound reproduction," J. Audio Eng. Soc. 47, 583-595 (1999).

${ }^{7}$ F. Asano, Y. Suzuki, and T. Sone, "Sound equalization using derivative constraints," Acustica 82, 311-320 (1996).

${ }^{8}$ A. O. Santillán, "Active equalization of the sound field in an extended region inside a room," Proc. 5th Int. Congress on Sound and Vibration, University of Adelaide, South Australia, December 15-18, 1997, edited by C. H. Hansen and G. Vokalek (International Institute of Acoustics and Vibration, South Australia, 1997), pp. 287-294.

${ }^{9}$ A. O. Santillán, "Experimental low-frequency sound equalization in an extended region of an enclosure using adaptive filters," Proc. 104th Convention Audio Eng. Soc., prep. No. 4755 (1998).

${ }^{10}$ A. O. Santillán and M. Lydolf, "Use of FIR filters to extend the frequency range in a test chamber for low-frequency noise and infrasound exposure," Proceedings of the 2001 International Congress and Exhibition on Noise Control Engineering (Inter-Noise 2001), The Hague, The Netherlands, August 27-30, 2001 (Nederlands Akoestisch Genootschap (NAG), The Netherlands, 2001), pp. 2179-2182.

${ }^{11} \mathrm{P}$. M. Morse and K. U. Ingard, Theoretical Acoustics (McGraw-Hill, New York, 1968)

${ }^{12}$ S. J. Elliott, I. M. Stothers, and P. A. Nelson, "A multiple error LMS
} 
algorithm and its application to the active control of sound and vibration," IEEE Trans. Acoust., Speech, Signal Process. 35, 1423-1434 (1987).

${ }^{13}$ F. Asano and D. C. Swason, "Sound equalization in enclosures using modal reconstruction," J. Acoust. Soc. Am. 98, 2062-2069 (1995).

${ }^{14}$ P. A. Nelson, F. Orduña-Bustamante, and H. Hamada, "Multichannel sig- nal processing techniques in the reproduction of sound," J. Audio Eng. Soc. 44, 973-989 (1996).

${ }^{15}$ A. O. Santillán, M. Uchiyama, and M. Tohyama, "Sound field control in a rectangular room at low frequencies to simulate the sound radiated by a virtual source," Technical Reports IEICE, 98, 53-60 (1999). 